\title{
Explorations in the social contagion of memory
}

\author{
MICHELLE L. MEADE and HENRY L. ROEDIGER III \\ Washington University, St. Louis, Missouri
}

\begin{abstract}
Four experiments examined social influence on the development of false memories. We employed the social contagion paradigm: A subject and a confederate see scenes and then later take turns recalling items from the scenes, with the confederate erroneously reporting some items that were not present in the scenes; on a final test, the subject reports these suggested items when instructed to recall only items from the scenes. The first two experiments showed that the social contagion effect persisted when subjects were explicitly warned about the possibility that confederates' responses might induce false memories and when they were tested via source-monitoring tests that explicitly gave the choice of attributing suggested items to the other person. Levels of false recall and recognition increased with the number of times the misleading information was suggested (Experiment 3), and subjects were more likely to incorporate the erroneous responses of an actual confederate on a recognition/source test as compared with those of a simulated confederate (Experiment 4). Collectively, the data support the claim that false memories may be transmitted between people and reveal critical factors that modulate the social contagion of memories.
\end{abstract}

False memories occur when people remember events differently from the way they actually occurred, or when they remember events that never happened. The occurrence of false memories has been experimentally confirmed in contexts ranging from simulated experiments on eyewitness testimony (e.g., Loftus \& Palmer, 1974), to learning lists of words (e.g., Roediger \& McDermott, 1995), to recall of prose stories (Bartlett, 1932), to remembering implications of sentences (Brewer, 1977), among many other techniques. Generally, these studies investigate the occurrence of false memories in individuals tested in isolation, as they reflect privately on events from their past. The study of the degree to which people remembering in groups mutually influence each other has been relatively neglected, even though people often remember experiences together as they attempt to assist each other in an effort to re-create a past event, or simply when they reminisce about old times.

The experimental study of social influences on memory is relatively recent, although not without important historical precursors (see Weldon, 2000, for a review and analysis). Before describing the present research, we briefly review past attempts to experimentally study social influences on development of false memories. To presage our own research: The social contagion paradigm developed by Roedi-

Experiments 1-3 were based on a master's thesis by M.L.M. conducted under supervision of H.L.R. We thank David A. Balota and Alan J. Lambert, the other members of the committee, for their advice and comment. We also thank Patrick O. Dolan, David A. Gallo, Larry L. Jacoby, Stephanie C. Lee, Elizabeth J. Marsh, and Kathleen B. McDermott for discussions of this work, and Daniel Wright and D. Stephen Lindsay for helpful comments. Correspondence should be addressed to M. L. Meade, Department of Psychology, Box 1125, One Brookings Drive, Washington University, St. Louis, MO 63130-4899(e-mail: mlmeade@ artsci.wustl. edu). ger, Meade, and Bergman (2001) and explored here melds two famous means of examining social influence, the technique used by Asch (e.g., Asch, 1956) to study conformity and the eyewitness misinformation paradigm developed by Loftus (e.g., Loftus \& Palmer, 1974). We briefly review each area of inquiry.

Binet (1900, as cited in Fancher, 1996; Whipple, 1909) was apparently the first researcher to systematically examine conformity and the influence of other people's suggestions on the behavior of individual children. He developed a line judgment task in which children were asked to judge whether a line was the same length as several comparison lines. Binet discovered that although children could successfully perform this simple line judgment task in isolation, they would respond with incorrect answers if Binet suggested that their own answers were wrong, or if other children (working as confederates) responded aloud with the incorrect answer before the subjects were able to respond. More than 50 years later, the stimuli and technique used by Binet were incorporated into the classic study of conformity conducted by Solomon Asch (1956), who found similar effects of conformity with adults.

Past research has identified both public and private types of conformity (Allen, 1965; Kelman, 1961). Public conformity is the type evident in the Asch (1956) study, where subjects altered their responses to be consistent with the responses of the rest of the group, even though they were aware that they were providing incorrect answers. Private conformity occurs when subjects have been so influenced by their social environments that they come to believe that the incorrect answer is actually true. The social contagion paradigm described here focuses on the processes involved in private conformity. In this sense, the current research differs from many of the aforementioned studies where conformity is public in nature. 
The literature on false memory is voluminous(see Roediger, 1996, Roediger \& McDermott, 2000, and Schacter, 1995 , for reviews). The false memory paradigm most relevant to the present work is the eyewitness or misinformation technique developed by Elizabeth Loftus and her associates (e.g., Loftus, Miller, \& Burns, 1978; Loftus \& Palmer, 1974; see Ayers \& Reder, 1998, for a review). In Loftus et al. (1978), subjects viewed slides depicting a traffic accident at an intersection with a stop sign (in one condition). They were later presented with information about a traffic sign in the scene that was consistent (stop sign), inconsistent (yield sign), or neutral (traffic sign) with respect to the nature of the sign in the initially presented scene. On a later forced-choice recognition test, subjects who had received inconsistent information were significantly less accurate than those who had received neutral information, whereas those who had received consistent information responded most accurately.

Similar results have been obtained in other contexts as well, such as misinformation effects resulting from forcedchoice recognition tests (Schreiber \& Sergent, 1998) and subjects' repeated retrieval on cued-recall tests (Roediger, Jacoby, \& McDermott, 1996). The reports of people in eyewitness testimony studies are often influenced by leading questions asked after the event (e.g., Ceci \& Bruck, 1995; Loftus \& Palmer, 1974). In all of these contexts, the misleading information changes subjects' reports about the original event.

In the typical eyewitness/misinformation experiment, there is no direct social presence. That is, misleading information is typically delivered in an impersonal written form, either embedded in questions that are assumed to have come from the experimenter or in a detailed narrative from a supposed observer of the original event. In either case, subjects may expect that information placed in the questions or narrative is correct, because either the experimenter presented the information, or a person with a remarkable knack for remembering fine details did. Therefore, the usual eyewitness experiment has only an implied social aspect, because no other people assumed to be in a position similar to the subject are physically present and trying to recollect the scene. Although exceptions do exist (see Ceci, Ross, \& Toglia, 1987; Shaw, Garven, \& Wood, 1997), the absence of actual other people separates misinformation studies of false memory from most studies of conformity. In the social contagion paradigm described below, the misleading information is provided to subjects directly by confederates who seem, to the actual subject, to be in the same situation during recall as is the subject.

Several recent studies have examined memory for events after exposure to misinformation presented by another person. However, it is unclear whether these studies examined changes in the subjects' beliefs about their reported memories (akin to private conformity) or rather influenced public conformity. Betz, Skowronski, and Ostrom (1996) asked subjects to read a prose passage and answer questions about it on a series of later memory tests. After being presented with information that hypothetical people had answered consistently or inconsistently with the prose passage, a significant number of subjects changed the answers they had originally chosen on the memory test to be consistent with the answers they believed other people had given. However, it is unclear whether subjects' beliefs about what was in the prose passage actually changed, or whether they were simply conforming to the responses of others (e.g., "I don't really remember the item from the passage, but the other person said it was there, so I guess it was").

Luus and Wells (1994) provided further evidence that the hypothetical responses of others may influence subjects' memory reports. When asked to identify a suspect from a photo lineup, subjects were less confident in their responses when they were informed by the experimenter that the other subject (who had been physically present during the staged crime scene, but not during the identification) had chosen a different suspect than the one identified by the subject. When subjects were informed that the other subject had chosen the same suspect as they had, confidence in their answers increased. In the Betz et al. (1996) study, the consistent or inconsistent information was delivered by the experimenter, and in the Luus and Wells study, by a police officer, so the source of misinformation could be considered to have high credibility and associated demand characteristics for compliance. Hoffman, Granhag, Kwong See, and Loftus (2001) directly manipulated the credibility of an implied social presence and found that subjects' reality-monitoring decisions were more heavily influenced by a high credibility source (a graduate student) than by a low-credibility source (flipping a coin).

Schneider and Watkins (1996) and Wright, Self, and Justice (2000) relied on an actual social presence to introduce misinformation. These authors showed that the judgment of another subject/confederate responding just before the real subject affected the responses of the true subject on a memory test. However, subjects were required to respond immediately after the other subject/ confederate had responded and to respond aloud in the presence of the other subject/confederate and the experimenter. Once again, the observed changes could have been due to public conformity (just going along with the group) rather than (or in addition to) private change in belief/memory about the event in question. Schneider and Watkins argued that the confidence ratings subjects gave for each of their responses indicated the extent to which people believed in the accuracy of their given response. The confidence ratings were given publicly, however, and are thus also subject to expectancy biases and response conformity confounds. Wright et al. did allow private confidence ratings and found that subjects were less confident in their responses after having heard contradictory information from the confederate. Such a finding may suggest private acceptance of the confederate's earlier suggestions. Regardless of whether the changes in memory reports in the Schneider and Watkins and Wright et al. experiments were caused by public or private conformity, we believe the effects are interesting. In our studies, we wanted to determine the extent to which erroneous responses changed 
the private belief/recollection of the subjects following misinformation delivered by a confederate.

The most compelling evidence that incorrect information from anotherperson may influence private belief comes from a study by Shaw et al. (1997). They showed that after a 48-h delay, subjects' memories on a final-recall test still showed the influence of the confederate's previous report. Such data suggest that it may be possible for subjects to privately believe the information suggested by a confederate.

The social contagion paradigm introduced by Roediger et al. (2001) provides a technique to further examine social influences on individual recollection as opposed to public conformity. As originally developed, the technique involved 2 ostensible subjects reporting to the laboratory, where one was a confederate and the other was the actual subject. They saw six scenes of typical household vistas (e.g., a kitchen) with instructions to remember them for a later test. After viewing the scenes, the subjects took a collaborative recall test taking turns to recall out loud 12 items from each scene, six items for each subject. (Pilot testing showed that subjects could recall this many items from the scenes.) For three of the scenes, the confederate recalled all six items correctly (the recalled objects had appeared in the scene). However, for the other three scenes, two of the six items recalled by the confederate were incorrect. These incorrect responses supplied misinformation for the real subject by suggesting objects that had not in fact appeared in the scene. One of these suggested objects was typical of the scene (e.g, a toaster for the kitchen scene), whereas the other object was less typical (e.g., oven mitts). On a later test of subjects in isolation, they were asked to remember as many items as possible for each of the six scenes, being sure to recall only items that had been in the original scenes.

On the individual recall test, subjects recalled the items erroneously reported by the confederate more often than they recalled the items that had not been suggested by the confederate. Because the confederate's false memories seemed to infect the subject's memory, we referred to the effect as the "social contagion of memory." Further, this social contagion effect was greater when the suggested information was typical of the scene, although it occurred for both typical and atypical items. The effect is probably due to private acceptance of the misinformation rather than public conformity, because the test occurred after a delay following the confederate's suggestion, in private, and with instructions to recall only items that had been originally seen. This conclusion about private conformity/ memory change is examined further in the present series of experiments.

Two further findings from Roediger et al. (2001) are of interest. First, when subjects assessed their recollections in the final test using Tulving's (1985) remember/know technique, the predominant response for the contagion (misinformation) items was for subjects to say that they knew the items had been in the scenes, but they did not remember them from the scenes. In addition, when the presentation time for the original scenes was varied (subjects studied the scenes for either 15 or $60 \mathrm{sec}$ each), the greater social contagion effect occurred at the faster rate. Apparently, the better the information is encoded, the less amenable it is to later false recollection, a finding that occurs in other paradigms as well (e.g., Gallo \& Roediger, 2002; McDermott \& Watson, 2001; Toglia, Neuschatz, \& Goodwin, 1999).

The purpose of the present series of four experiments was to pinpoint factors that modulate the social contagion phenomenon. We selected variables known to have an effect in other domains of false memory research to determine whether they would generalize to the social contagion paradigm, which is a hybrid of the conformity and misinformation paradigms. We deal with theoretical implications of our findings in the General Discussion, but the primary motivation of the research was explicitly empirical and methodological: Would variables known to affect development of false memories in other paradigms generalize to the social contagion paradigm? In addition, is the change observed in memory reports due to private or public conformity? Although we argued earlier that the social contagion effect is probably due to private acceptance of the suggested memories, the fact that these reports are accompanied by know responses rather than remember responses leaves the issue in some doubt. False memories are often accompanied by remember responses in some paradigms (e.g., Roediger \& McDermott, 1995), which implicates true "false remembering"- the remembering of events that did not occur. False know responses are more ambiguous in their meaning, but if subjects are following instructions typically given (see Tulving, 1985; but also see Gardiner, 1988, and Rajaram, 1993, for expanded instructions), then know responses should also indicate private rather than public conformity.

\section{EXPERIMENT 1}

Experiment 1 was designed to gain further evidence on the nature of false memories aroused in the social contagion paradigm by employing a source-monitoring test (Johnson, Hashtroudi, \& Lindsay, 1993). Source-monitoring tests give subjects the option of saying they remember an item only from the postevent suggestion; this option can help determine whether subjects believe that a contagion item occurred in the slides (as opposed to remembering it from the suggestion). In prior research with the misinformation paradigm, Lindsay and Johnson (1989; also see Lindsay, 1994) showed that the misinformation effect was greatly reduced or even eliminated on a source-monitoring test, a form of recognition test, so we wished to determine whether the same would be true of the social contagion paradigm. In addition, we wanted to determine whether the social contagion effect would survive a strong warning given to subjects just before their final test. The explicit warning against social influence is meant to reduce the experimental demand that may encourage conforming responses. Warnings have been shown to reduce but not eliminate false memories in other paradigms (e.g., Betz et al., 
1996; Gallo, Roberts, \& Seamon, 1997; Gallo, Roediger, \& McDermott, 2001; McDermott \& Roediger, 1998; Wright, 1993).

We expected that the social contagion effect would be reduced in Experiment 1, but that it would survive these more stringent test conditions involving explicit warnings and a source-monitoring test. However, because the misinformation effect is reduced by prior warnings (e.g., Weingardt, Toland, \& Loftus, 1994; Wright, 1993) and has been eliminated by a source-monitoring test in at least one study (Lindsay \& Johnson, 1989), it seemed possible that the social contagion effect might be entirely eliminated under the stringent conditions of this experiment.

\section{Method}

Subjects. The subjects were 37 Washington University undergraduates who participated in the experiment for partial fulfillment of a course requirement and who were tested individually. One subject was identified as being suspicious of the confederate, so we excluded his data from the analyses.

Design. The experiment consisted of a $2 \times 2 \times 2$ mixed design. Exposure to social contagion (contagion or no contagion) and the expectancy of the contagion items within a scene (high-expectancy or low-expectancy) were manipulated within subjects, whereas the instructions for the individual recall test (warning or no warning) were manipulated between subjects (18 per condition). The primary dependent variables were false recall and false recognition of the suggested items.

Materials. Materials included the six scenes (toolbox, bathroom, kitchen, bedroom, closet, and desk) used in Roediger et al. (2001) and available on the Web at www.psych.wustl .edu/memory/stimuli/scs. $\mathrm{htm}$. Each scene contained an average of 23.8 items that were either high- or low-expectancy (or typicality), as determined in a pilot study in which students listed items they expected to see when given the name of the scene (see the Appendix). Two high- and two lowexpectancy items were excluded from each slide so that these items could later be used as contagion items. Contagion (or misinformation) items are those that the confederates only suggested were present in the scene.

Additional materials included a multiplication filler task, individual recall sheets, and a final recognition test. The recognition test contained 18 previously studied items (three items from each of the six scenes), 12 potentially misleading items (the high- and lowexpectancy contagion items from each of the six scenes), and six unrelated, concrete items not included in any of the scenes.

Procedure. One subject and 1 confederate participated in each experimental session. Six slides of common household scenes were always presented in the following order: toolbox, bathroom, kitchen, bedroom, closet, and desk. Each scene was presented for $15 \mathrm{sec}$, and was verbally labeled as it appeared (e.g., "the toolbox scene," "the bathroom scene," etc.). Subjects were instructed to pay attention because they would be asked to remember items from each scene on a later memory test. After seeing all six slides, the subject and the confederate were given a filler task requiring them to solve multiplication problems for $4 \mathrm{~min}$.

Next, the subject and the confederate participated in a collaborative recall phase during which they alternated recalling items from each scene after being given the name of the scene. Recall of the scenes occurred in the same order in which the scenes had been presented, and the experimenter recorded each recalled item. The subject and confederate continued this task until they had each recalled six items from each of the six scenes. During this collaborative recall phase, the confederate recalled items that had occurred in the scenes (correct items) as well as items that had not actually been present in the scenes (contagion items). Specifically, for three of the six scenes, the confederate recalled six items that had actually been present in the scenes, and for the other three scenes, the confederate recalled four items that had actually been present as well as two items that had not been in the scene. One of the false or contagion items recalled by the confederate was a high-expectancy item and one was a low-expectancy item. The confederate always recalled the high-expectancy contagion item in the fourth position and the lowexpectancy contagion item in the sixth position. (A pilot experiment not reported here counterbalanced these positions and showed no difference.) The scenes with contagion items were counterbalanced so that the contagion items for each scene occurred for half the subjects.

Throughout the experimental session, the confederates complied to a carefully scripted procedure. Prior training had allowed them to memorize a set of responses for each scene and to learn to "recall" the items in a manner similar to how an average subject might recall items. Confederates were further instructed to match the subject's level of recall, so that the confederate did not appear to know too much or too little and thus arouse suspicion. A list of alternative responses for each scene was also memorized. Confederates reported these alternative responses when the subject recalled one of the confederate's items before the confederate (this happened only twice for the contagion items).

Shortly after the collaborative recall phase, the subject and the confederate were asked to complete an individual recall test; they were placed in separate rooms and asked to recall items from each of the scenes on their own. (At this point, the confederate did not actually recall the scenes, but merely read while in the individual testing room.) All subjects were told to recall as many items as they could remember from each of the scenes, but half the subjects were also warned that the confederate might have made mistakes by reporting things that were not actually in the scenes. They were instructed to write down only items that they were sure had actually appeared in the scenes and were given 2 min (for each scene) to write down their responses in any order. For each written item, subjects were next asked to indicate whether they remembered or knew that the item had occurred in the scenes. This remember/know distinction was based on the instructions of Gardiner (1988), Rajaram (1993), and Tulving (1985). Remember responses indicated that subjects consciously recollected the item from the scene, whereas know responses indicated that subjects had no specific recollection of the item, but were confident that the item had occurred in the scene. Because subjects were instructed to write down only items that they thought were from the scene, we did not include a "guess" judgment option in addition to the remember and know judgments. After the 2 min had passed, the experimenter opened the door and handed the subject a new sheet of paper with instructions to recall items from the next scene. The door to the confederate's room was also opened every 2 min to give subjects the illusion that the confederate was undergoing an identical procedure.

Finally, subjects were presented with a 36-item recognition/ source-monitoring test that required them to identify the source of each item on the test. As described previously, half the items were targets and half were lures. Twelve lures represented the contagion items (six having been suggested by the confederate and six serving as controls). Subjects were asked to indicate whether they recognized each item as having been in the scene (scene only), as having been mentioned by the other subject (other subject only), as having been in the scene and having been mentioned by the other subject (both sources), or not having been in the scene and not mentioned by the other subject (neither source). If subjects remembered saying the item themselves, they were instructed to check the "scene-only" box if they thought it had appeared in one of the scenes, and to check the "neither" box if it had not appeared in one of the scenes. Subjects were given as much time as they needed to complete the final recognition task, but it generally took less than $15 \mathrm{~min}$. They were then thanked for their participation and fully debriefed. 


\section{Results and Discussion}

The false recall results are shown in Table 1 separately for subjects who received warnings and for those who did not, as well as for high- and low-expectancy items. The top three rows in the table show overall recall, remember, and know responses for the suggested contagion items (the high- and low-expectancy items suggested by the confederate), the next three rows do so for control items (the high- and low-expectancy items when not suggested by the confederate), and the last three rows report the difference between the top two sets of rows and therefore indicate the social contagion effect. Within each grouping of three, remember and know responses sum to total recall; differences are due to rounding error. Results judged statistically significant meet the criterion of $p<.05$.

The results reveal greater levels of false recall for contagion items than for control items, especially for nonwarned subjects, replicating Roediger et al. (2001). A 2 (contagion or control) $\times 2$ (high- or low-expectancy) $\times 2$ (warning or no warning) ANOVA on the mean proportion of recalled items revealed a main effect of contagion $\left[F(1,34)=19.71, M S_{\mathrm{e}}=0.08\right]$. Subjects falsely recalled a significantly greater proportion of the items when these had been suggested by the confederate $(M=.26)$, relative to when they had not been suggested by the confederate $(M=.06)$. This is the social contagion effect.

There was also a significant main effect of expectancy, again replicating Roediger et al. (2001) $[F(1,34)=14.71$, $\left.M S_{\mathrm{e}}=0.04\right]$. Subjects were more likely to falsely recall high-expectancy contagion items $(M=.23)$ than lowexpectancy contagion items $(M=.10)$. These means are collapsed across contagion and control items in the warning and no-warning conditions shown in Table 1. Greater recall for high- than for low-expectancy items even in the control condition replicates past studies, which have shown high levels of false recall for highly associated items (e.g., Brewer \& Treyens, 1981; Miller \& Gazzaniga, 1998; Roediger \& McDermott, 1995).

The main effect of warning was also significant $\left[F(1,34)=6.18, M S_{\mathrm{e}}=.05\right]$; the contagion effect was sig- nificantly reduced in the warning condition $(M=.11)$ relative to the no-warning condition $(M=.30)$. The effect of warning interacted significantly with the contagion effect $\left[F(1,34)=4.91, M S_{\mathrm{e}}=0.08\right]$, indicating that warning significantly reduced the number of contagion items recalled ( $M=.36$ with no-warning and .17 with warning), but had no effect on the control items, perhaps due to a floor effect $(M=.06$ in both the no-warning and warning conditions). Therefore, the social contagion effect was relatively greater in the no-warning than in the warning condition, but survived even the stringent warning $[t(17)=$ 2.15$, SEM $=.05]$.

The effect of warning on recall of the contagion items could have been due to a general criterion shift. Perhaps subjects recalled fewer contagion items in the warning condition because they edited out most responses the confederate said, both those in the scenes and those only suggested by the confederate. Table 2 shows the mean proportion of items in the warning and no-warning conditions that were recalled by subjects on the individual recall test and that had previously been recalled on the collaborative recall test by the subjects themselves, by the confederate, or by neither the subject nor the confederate. As the table shows, the warning had no significant effect on the items subjects themselves had previously recalled on the collaborative recall test. However, the warning did influence subjects' recall of items previously recalled by the confederate, even the veridical ones that had appeared in the scene $\left[F(1,34)=16.41, M S_{\mathrm{e}}=0.03\right]$. Specifically, subjects were less likely to recall correct items previously recalled by the confederate when they were given a warning about possible errors by the confederate $(M=.37)$ relative to when they were not given a warning $(M=.47)[t(34)=$ $2.39, S E M=.04]$. The same pattern was observed for the contagion items as well. Of contagion items previously recalled by the confederate, subjects recalled a mean of .17 when given a warning relative to a mean of .36 when not given a warning $\left[t(34)=2.25, M S_{\mathrm{e}}=0.09\right]$. (These means are collapsed across high- and low-expectancy items.) The warning did reduce the number of items recalled by sub-

Table 1

Experiment 1: Mean Proportion of False Recall and Remember or Know Responses for High- and Low-Expectancy Items Recalled

\begin{tabular}{|c|c|c|c|c|c|}
\hline \multirow{2}{*}{$\begin{array}{l}\text { Response } \\
\text { Type }\end{array}$} & \multicolumn{2}{|c|}{ No Warning } & \multicolumn{2}{|c|}{ Warning } & \multirow[b]{2}{*}{$M$} \\
\hline & Low Expect & High Expect & Low Expect & High Expect & \\
\hline \multicolumn{6}{|c|}{ Contagion Items } \\
\hline Recall & .26 & .46 & .09 & .24 & .26 \\
\hline Remember & .07 & .17 & .00 & .09 & .08 \\
\hline Know & .19 & .29 & .09 & .15 & .18 \\
\hline \multicolumn{6}{|c|}{ Control Items } \\
\hline Recall & .04 & .09 & .00 & .11 & .06 \\
\hline Remember & .01 & .03 & .00 & .02 & .02 \\
\hline Know & .03 & .06 & .00 & .09 & .04 \\
\hline \multicolumn{6}{|c|}{ Difference } \\
\hline Recall & .22 & .37 & .09 & .13 & .20 \\
\hline Remember & .06 & .14 & .00 & .07 & .06 \\
\hline Know & .16 & .23 & .09 & .06 & .14 \\
\hline
\end{tabular}


Table 2

Experiment 1: Mean Proportion of Items Recalled by Subjects on the Individual Recall Test That had Been Previously Recalled on the Collaborative Test by the Subject, the Confederate (Both Correct and High- and Low-Contagion Items), or by Neither the Subject nor the Confederate

Subject Confederate Confederate Contagion Not Correct Correct High Expect Low Expect Recalled

\begin{tabular}{llllll}
\hline No warning & .80 & .47 & .46 & .26 & .11 \\
Warning & .80 & .37 & .24 & .09 & .05 \\
Difference & .00 & .10 & .22 & .17 & .06 \\
\hline
\end{tabular}

jects that had been previously recalled by the confederate, and this reduction did not vary between correct and contagion items [the interaction between correct or contagion items previously recalled by the confederate and warning was not significant, $\left.F(1,34)=1.37, M S_{\mathrm{e}}=0.03, p>.05\right]$. Therefore, the reduction in the contagion effect seems to have been at least partly due to a general criterion shift, although numerically, the warning effect was greater for contagion items $(M=.20)$ than for control items $(M=$ .10 ), so a more powerful design (one with more observations) might have revealed a significant effect.

For each item on the recall test, subjects indicated whether they remembered the item as having occurred in the scenes as opposed to knowing it was there (Tulving, 1985). Table 1 presents the mean proportion of remember and know responses for contagion and control conditions and their difference. For contagion items, subjects were more likely to give a know judgment that a suggested item had been in the scene $(M=.18)$ than they were to give a remember judgment that the contagion item had been in the scene $(M=.08)[t(17)=2.01, S E M=.05]$. This difference was not reliable in the control condition $[M=.04$ for know responses and $M=.02$ for remember responses, $t(17)=1.31, S E M=.02, p>.05]$, perhaps because of a floor effect. This pattern-more know than remember responses after a confederate has suggested the itemsreplicates the findings of Roediger et al. (2001).

Remember and know responses were analyzed separately to determine how they related to contagion, expectancy, and warning. For the remember responses, a 2 (remember responses for contagion or control items) $\times 2$ (high- or low-expectancy) $\times 2$ (warning or no warning) ANOVA revealed a significant main effect of contagion items $\left[F(1,33)=8.12, M S_{\mathrm{e}}=0.03\right]$ and expectancy $\left[F(1,33)=5.68, M S_{\mathrm{e}}=0.02\right]$. Subjects were more likely to give remember judgments for suggested (contagion) items than for control items, and high-expectancy contagion words were more likely to be remembered than lowexpectancy words. Therefore, false remembering (in Tulving's, 1985, sense) did occur and was magnified by social contagion.

For know responses, significant main effects were found for contagion $\left[F(1,33)=21.40, M S_{\mathrm{e}}=0.74\right]$ and for expectancy $\left[F(1,33)=10.00, M S_{\mathrm{e}}=0.02\right]$. As for remember responses, know responses were significantly greater for contagion than for control items, and for high- expectancy than for low-expectancy items. In addition, there was a significant interaction between warning and contagion $\left[F(1,33)=5.05, M S_{\mathrm{e}}=0.03\right]$; thus the proportion of know responses was greater in the no-warning condition than in the warning condition.

Subjects' performance on the recognition/sourcemonitoring test is shown in Table 3, which shows performance for contagion and control items as a function of the warning and no-warning conditions in the four possible response categories on the source-monitoring test (seen in the slide, seen in the slide and spoken by the other subject, only spoken by the other subject, or neither seen in the slide nor spoken by the other subject). For contagion items, the correct response was "confederate only"; for control items, "neither slide nor confederate" was the correct response. We defined false recognition on the recognition/ source-monitoring test as the proportion of items subjects recognized as having occurred in the scene (slide only) plus the proportion of items that subjects recognized as having occurred in the scene and as having been suggested by the confederate (both slide and confederate). In both of these conditions, subjects judged the items as having occurred in the scenes even though they did not. False recognition is shown on the third row of Table 3. Subjects falsely recognized a mean proportion of .56 of the suggested items as having actually appeared in the scene relative to a mean proportion of .33 of those same items that had not been suggested by the confederate, a statistically significant difference $[t(35)=3.57, S E M=.06]$. This finding indicates that the social contagion effect occurred on the recognition source-monitoring test.

Interestingly, subjects actually attributed more control items $(M=.30)$ than contagion items $(M=.11)$ to the slide only $[t(35)=5.39, S E M=.04]$. The slide-only responses of the subjects in the contagion condition were probably reduced by their recollection of the other subject recalling the item, so they assumed the item had been both seen and heard. In addition, the high baseline rate of the control items $(M=.30)$ could be related to the general associative strength between the contagion items and the scenes. Because the contagion items are so strongly associated to the scenes, subjects may falsely remember them as having appeared in the scene (Brewer \& Treyens, 1981; Miller \& Gazzaniga, 1998). Nevertheless, the high levels of false recognition obtained in Experiment 1 demonstrate that the social contagion paradigm may alter subjects'

Table 3

Experiment 1: Mean Proportion of False and Veridical Source Judgments for Contagion and Control Items

\begin{tabular}{lccccccc}
\hline & \multicolumn{3}{c}{ Contagion Items } & & \multicolumn{3}{c}{ Control Items } \\
\cline { 2 - 3 } & NW & W & $M$ & & NW & W & $M$ \\
\hline Slide only & .10 & .12 & .11 & & .29 & .31 & .30 \\
Both slide and confederate & .51 & .39 & .45 & & .03 & .02 & .03 \\
$\quad$ Total false recognition & .61 & .51 & .56 & .32 & .33 & .33 \\
Confederate only & .34 & .36 & .35 & .02 & .03 & .02 \\
Neither slide nor confederate & .05 & .13 & .09 & .66 & .64 & .65 \\
\hline
\end{tabular}

Note-NW, no-warning condition; W, warning condition; $M=$ Mean collapsed across warning and no-warning conditions. 
memories for the original scenes. Subjects came to believe (via the confederate's suggestion) that objects that were only suggested had in fact been present in the scenes.

Recognition responses were further examined in relation to prior recall performance. Conditional analyses revealed very little difference between recognition of items previously recalled and items not previously recalled, although subjects appeared slightly more likely to recognize an item that had been previously recalled. The conditional analyses suffered from a small and unequal number of subjects and items in each condition, and are thus mentioned only to note that the same general trends were obtained. An experimental test of the influence of prior recall on recognition is provided in Experiment 2.

In terms of correct performance on items actually studied, subjects recalled a mean proportion of .30 items across the six scenes and correctly recognized a mean proportion of .71 across the six scenes. The proportions of veridical recall are based on an average of 23.8 items that could potentially be recalled from each scene. Correct recall was affected by warning: Subjects who were given a warning recalled a significantly lower proportion of items $(M=.27)$ than subjects who did not receive a warning $(M=.32)[t(34)=2.76, S E M=.02]$. However, warning had no reliable effect on correct recognition $(M=.73$ in the no-warning condition relative to $M=.70$ in the warning condition) $[t(34)=1.00, S E M=.03, p>.05]$.

\section{EXPERIMENT 2}

The purpose of Experiment 2 was to examine performance on the recognition/source-monitoring test with and without prior recall and also to extend Roediger et al.'s (2001) research on presentation rate. Experiment 1 revealed that on the final recognition test, subjects generally attributed the source of the contagion items to the scenes. However, the final recognition test always followed a recall test, and prior recall may have induced the source confusion that led to false memories (see Roediger et al., 1996). Our claim that subjects' recollections of the scenes are actually changed as a result of the confederate's suggestions would be strengthened by additional evidence indicating the same outcome when the recognition test is not confounded by prior recall.

In addition, we asked in Experiment 2 whether presentation rates faster than $15 \mathrm{sec}$ would increase the social contagion effect, as occurred when the rate was increased from 60 to $15 \mathrm{sec}$ per scene in Roediger et al. (2001). We expected that with poorer encoding of the original items, the social contagion effect would be even greater because subjects may rely more on others when remembering poorly encoded scenes (an idea consistent with Deutsch \& Gerard's, 1955, hypothesis of informative social influences), but this prediction is tempered by prior work showing an inverted $\mathrm{U}$-shaped function of presentation rate and false memories in other paradigms. Using the Deese-Roediger-McDermott (DRM) associative word list paradigm, McDermott and Watson (2001) found low rates of false recall with very fast (less than half a second) and very long $(3$ or $5 \mathrm{sec})$ presentation rates, relative to intermediate rates $(1 \mathrm{sec})$. Therefore, speeding the rate of scene presentation in the social contagion paradigm may not necessarily increase the level of false recall.

\section{Method}

Subjects. The subjects were 48 Washington University undergraduates who were tested individually and who participated in the experiment as partial fulfillment of a course requirement.

Design. The experiment consisted of a $2 \times 2 \times 2 \times 2$ mixed design. Exposure to social contagion (contagion or no contagion) and the expectancy of the contagion items (high-expectancy or lowexpectancy) were manipulated within subjects, whereas the presentation rate (5 or $15 \mathrm{sec})$ and prior recall (recall or no recall) were manipulated between subjects. The primary dependent variables were false recall and false recognition of the suggested items.

Materials. The same materials used in Experiment 1 were also used in Experiment 2. Additional materials included a filler task involving recall of items from semantic categories.

Procedure. The procedure was identical to that of Experiment 1 except for the presentation rate of the scenes, whether or not the individual recall test occurred prior to the recognition test, and the absence of a warning condition.

Experiment 2 varied with respect to the type of individual recall test given to subjects. The subjects in the prior-recall condition were given an individual recall test identical to the one used in Experiment 1 . The other half of the subjects (those in the no-prior-recall condition) were given six different semantic categories (U.S. presidents, European capitals, mountain ranges, impressionist artists, African countries, and professional football players) and had $2 \mathrm{~min}$ for each category to list as many members as possible. These semantic categories were administered simply to equate the time between collaborative recall and final recognition for the two groups.

\section{Results and Discussion}

The results of Experiment 2 nicely replicated those of Experiment 1: Significant levels of social contagion were obtained and the effect was again greater for high- than for low-expectancy items. Table 4 presents the mean proportion of high- and low-expectancy items that were falsely recalled when the scenes were presented for 5 and $15 \mathrm{sec}$. A 2 (contagion or control) $\times 2$ (high- or lowexpectancy) $\times 2$ (5- or 15 -sec presentation rate) ANOVA revealed a significant main effect of contagion $[F(1,22)=$ $\left.43.79, M S_{\mathrm{e}}=0.04\right]$. Subjects were more likely to falsely recall the items when the items had been suggested by the confederate $(M=.40$, collapsing across 5 and $15 \mathrm{sec})$ than when those same items had not been suggested by the confederate $(M=.14)$. The main effect of contagion shows that subjects incorporated the responses of the confederate into their recollections of the scenes.

There was also a significant main effect of expectancy $\left[F(1,22)=15.80, M S_{\mathrm{e}}=0.07\right]$. Subjects were more likely to incorporate the contagion items into their own memory reports when the items were high-expectancy $(M=.37$ collapsing across 5 and $15 \mathrm{sec}$ and contagion and control items) than when they were low-expectancy $(M=.16)$. As discussed earlier, greater levels of false recall for highexpectancy items may occur because high-expectancyitems are more strongly associated to the scene. The main effect of presentation rate was not significant $\left[F(1,22)=.02, M S_{\mathrm{e}}=\right.$ 
Table 4

Experiment 2: Mean Proportion of False Recall and Remember or Know Responses for Low- and High-Expectancy Words Recalled When Scenes Were Presented to Subjects for 5 and $15 \mathrm{Sec}$

\begin{tabular}{|c|c|c|c|c|c|}
\hline \multirow{2}{*}{$\begin{array}{c}\text { Response } \\
\text { Type }\end{array}$} & \multicolumn{2}{|c|}{$5 \mathrm{Sec}$} & \multicolumn{2}{|c|}{$15 \mathrm{Sec}$} & \multirow[b]{2}{*}{$M$} \\
\hline & Low Expect & High Expect & Low Expect & High Expect & \\
\hline \multicolumn{6}{|c|}{ Contagion Items } \\
\hline Recall & .19 & .53 & .33 & .53 & .40 \\
\hline Remember & .06 & .11 & .04 & .07 & .07 \\
\hline Know & .13 & .42 & .29 & .46 & .33 \\
\hline \multicolumn{6}{|c|}{ Control Items } \\
\hline Recall & .08 & .24 & .03 & .19 & .14 \\
\hline Remember & .00 & .06 & .00 & .03 & .02 \\
\hline Know & .08 & .18 & .03 & .16 & .12 \\
\hline \multicolumn{6}{|c|}{ Difference } \\
\hline Recall & .11 & .29 & .30 & .34 & .26 \\
\hline Remember & .06 & .05 & .04 & .04 & .05 \\
\hline Know & .05 & .24 & .26 & .30 & .21 \\
\hline
\end{tabular}

$0.07, p>.05]$. Subjects were no more likely to falsely recall the contagion items when they had been presented with the slides for 5 or $15 \mathrm{sec}$. No interactions were significant.

In terms of remember and know responses, there was again a significant difference between the proportion of remember and know responses given on the individual recall test. Table 4 presents the mean proportion of remember and know responses for the contagion and control conditions and their difference. As in Experiment 1, subjects were more likely to say they knew that the suggested (contagion) items had been in the scene $(M=.33)$ than they were to say that they actually remembered the items from the scenes $(M=.07)[t(23)=4.34$, SEM $=.06]$. This same pattern was obtained when subjects falsely recalled these items when they had not been suggested by the confederate $(M=.12$ for know responses and $M=.02$ for remember responses) $[t(23)=3.09, S E M=.03]$.

As in Experiment 1, remember and know responses were analyzed separately to determine how they related to contagion, expectancy, and presentation rate. When remember responses were compared across conditions, a 2 (remember responses for contagion or control items) $\times 2$ (high- or low-expectancy) $\times 2$ (5- or 15 -sec presentation rate) ANOVA revealed no significant main effects or interactions (perhaps due to floor effects). The pattern of results did generally resemble that for remember responses in Experiment 1, albeit at lower levels.

Know responses were analyzed across conditions in the same manner. In contrast to the analysis of remember responses, that of know responses revealed a significant main effect of contagion $\left[F(1,22)=20.36, M S_{\mathrm{e}}=0.05\right]$. Subjects were more likely to give a know judgment to the items that had been suggested by the confederate $(M=$ .33) than to those items when they had not been suggested by the confederate $(M=.12)$. Further, subjects were more likely to say they knew that high-expectancy items had been in the scenes $(M=.31)$ than low-expectancy items $(M=.13)\left[F(1,22)=11.79, M S_{\mathrm{e}}=0.02\right]$. No significant main effect of presentation rate was observed $[F(1,22)=$ $\left..22, M S_{\mathrm{e}}=0.08, p>.05\right]$, nor were there any significant interactions between presentation rate and other variables (although the data suggest that know responses for contagion items were relatively higher in the 15 -sec condition than in the 5-sec condition).

Table 5

Experiment 2: Mean Proportion of False and Veridical Source Judgments When Subjects Were Presented With Each Scene for 5 and 15 Sec

\begin{tabular}{|c|c|c|c|c|}
\hline & \multicolumn{2}{|c|}{$5 \mathrm{Sec}$} & \multicolumn{2}{|c|}{$15 \mathrm{Sec}$} \\
\hline & Contagion Items & Control Items & Contagion Items & Control Items \\
\hline \multicolumn{5}{|c|}{ With Prior Recall } \\
\hline Slide only & .13 & .46 & .13 & .31 \\
\hline Both slide and confederate & .49 & .06 & .47 & .07 \\
\hline Total false recognition & .62 & .52 & .60 & .38 \\
\hline Confederate only & .28 & .01 & .29 & .03 \\
\hline Neither slide nor confederate & .10 & .47 & .11 & .60 \\
\hline \multicolumn{5}{|c|}{ With No Prior Recall } \\
\hline Slide only & .21 & .54 & .07 & .36 \\
\hline Both slide and confederate & .56 & .06 & .60 & .08 \\
\hline Total false recognition & .77 & .60 & .67 & .44 \\
\hline Confederate only & .19 & .01 & .26 & .03 \\
\hline Neither slide nor confederate & .04 & .39 & .07 & .53 \\
\hline
\end{tabular}


The source-monitoring data shown in Table 5 again indicate a significant effect of social contagion. Further, the effect occurred both in conditions with a prior recall test $(M=.16$ effect $)$ and without a prior recall test $(M=.20$ effect). (These data are averaged over the two presentation rates). A 2 (contagion or control) $\times 2$ (5- or 15 -sec presentation rate) $\times 2$ (prior recall or no prior recall) ANOVA revealed a significant main effect of contagion $[F(1,44)=$ $\left.13.90, M S_{\mathrm{e}}=0.05\right]$. False recognition of items suggested by the confederate ( $M=.66$ collapsing across presentation rate and prior recall conditions) was significantly greater than false recognition of those same items when they had not been suggested $(M=.48)$. As in Experiment 1 , subjects incorporated the responses of the confederate into their own memory reports and attributed the source of the contagion items to the scenes. Importantly, there was no main effect of prior recall $[F(1,44)=1.23$, $\left.M S_{\mathrm{e}}=0.14, p>.05\right]$. Whether or not subjects had previously recalled items from the scene, their performance on the final recognition test was about the same. Therefore, the results of Experiment 1 were replicated twice and those results do not seem to be due to prior recall. The social contagion effect occurs on a source-monitoring test whether or not this test is preceded by a cued-recall test on the relevant material.

There was no significant main effect of presentation rate on recognitionin the source-monitoring test $[F(1,44)=$ $\left.1.41, M S_{\mathrm{e}}=0.14, p>.05\right]$. Final recognition performance did not vary in relation to how long subjects were originally presented with each scene. As in Experiment 1, subjects actually attributed a greater proportion of control items than contagion items to having occurred in the scene only. Again, this result is most likely attributable to the high associative strength that exists between the contagion items and the items in the scenes (e.g., Miller \& Gazzaniga, 1998; Roediger \& McDermott, 1995).

Finally, subjects correctly recalled a mean proportion of .29 items from the six scenes. The range of veridical recall was .20 to .42 across scenes. The mean proportion of correct recognition was .81 and ranged across the six scenes from .70 to .91 .

\section{EXPERIMENT 3}

Experiment 3 was designed to determine whether the social contagion effect obtained in Experiments 1 and 2 could be enhanced with the number of times the false information was presented. Research conducted on both conformity (Asch, 1956) and the misinformation paradigm (Mitchell \& Zaragoza, 1996; Zaragoza \& Mitchell, 1996) suggests that increased exposures or repetition will increase the likelihood that subjects will conform or falsely remember information. To examine the relationship between repetition and false responding in the social contagion paradigm, we created contagion protocols of hypothetical subject/confederates (as did Betz et al., 1996), which allowed us to suggest contagion items to naive subjects multiple times. The contagion protocols were handwritten on sheets of paper to resemble the written responses of other subjects. We relied on the implied social presence of others via the protocols to introduce the contagion information rather than using the actual confederates because having a single confederate recall the same contagion item repeatedly or having many confederates repeat the same item might arouse suspicion in subjects. In addition, introducing the misinformation through an implied social presence allowed us to determine whether social contagion could be obtained in this indirect manner. Of course, the work of Betz et al. led us to expect positive results.

\section{Method}

Subjects. The subjects were 15 Washington University undergraduates, who participated in the experiment for partial fulfillment of a course requirement and who were tested individually.

Design. The experiment consisted of a $3 \times 2$ within-subjects design. The number of times subjects were exposed to social contagion (zero, one, or four times) was manipulated within subjects. In this case, the zero-presentation condition served as the control condition. The expectancy of the contagion items (high or low) was also manipulated within subjects. The dependent variables were false recall and false recognition of the contagion items.

Materials. The same materials used in Experiments 1 and 2 were also employed in Experiment 3. Additional materials included contagion protocols. There were five contagion protocols for each of the six scenes (for a total of 30) that were presented to the subjects as recall tests of subjects who had previously been in the study. In reality, the experimenter had constructed the protocols to include items that had been recalled by many subjects in Experiments 1 and 2 as well as items that had only been recalled by a few individuals in Experiments 1 and 2 . The number of items on each of the protocols (6-13) was representative of the range of the number of items actually recalled by subjects. For each scene, zero, one, or four of the protocols contained the contagion items. Both high- and lowexpectancy contagion items were included. The protocols were handwritten by five people to represent the responses of 5 subjects.

Procedure. Experiment 3 followed the same general procedure as Experiments 1 and 2. Subjects viewed each of the slides for $15 \mathrm{sec}$ and completed the 4-min filler task, as in Experiments 1 and 2. Then they were asked to complete an individual recall test in which they were given 2 min to recall as many items as possible from each scene and to make remember/know judgments for each of their responses. After this initial recall test, subjects were presented with the contagion protocols. They were told that the experimenter was interested in how well people could assess their own memories. To this end, subjects had $15 \mathrm{sec}$ to examine each protocol and determine whether they did a better or worse job than the protocol subject in recalling items from each of the scenes. Subjects were thus to compare their own performance on the initial recall test with the performance of the protocol subject. They made this comparison from memory since the subjects' own original responses had been removed. Subjects were given a response sheet, and for each protocol (five for each of the six scenes), they were instructed to simply place a check under the heading "me" if they had done a better job than the protocol subjects and to place a check under the heading "other subject" if the protocol subject had done a better job. To ensure that subjects actually encoded the items on the protocols, they were instructed to circle the words on each protocol that they themselves remembered having recalled on the initial recall test. They followed this procedure for each of the five protocols.

Subjects were then given a second individual recall test that was identical to the first recall test. They were again allotted $2 \mathrm{~min}$ for each scene that was cued by its name, and they were asked to make 
remember/know judgments for all responses. They were told to try to be as accurate as possible in recalling only items that had appeared in the scenes. Finally, subjects were given the same recognition/ source-monitoring test used in Experiments 1 and 2.

\section{Results and Discussion}

Table 6 shows the false recall results on the second test following zero, one, or four exposures of the contagion items in hypothetical subjects' protocols, for both highand low-expectancy items. False recall increased with the number of times the contagion items were presented to the subjects. A 3 (zero, one, or four presentations) $\times 2$ (highor low-expectancy) ANOVA revealed a significant main effect of the number of times the contagion information was presented $\left[F(2,28)=21.56, M S_{\mathrm{e}}=0.04\right]$. The social contagion effect was greater when the contagionitems had been suggested on the protocols four times $(M=.32)$ as opposed to one time $(M=.09)$. These means are collapsed across high- and low-expectancy items and subtract out the control condition $(M=.06)$. The difference between the levels of false recall when the contagion information was presented one time $(M=.15)$ and when it was presented zero times $(M=.06)$ was not significant $[t(14)=1.58, S E M=.05]$. Experiment 3 provided evidence that social contagion exists when the misinformation is introduced through an implied social presence rather than through a confederate, replicating Betz et al. (1996). However, the results differ statistically from those of the earlier experiments, possibly because the written protocols had less impact than an actual confederate would have (of course, the difference is in the same direction as in earlier experiments). In Experiments 1 and 2, the contagion item was presented only once and did have a significant effect, but the confederate spoke the item aloud during the initial joint test. This issue of the relative power of implied and actual social presence will be explored in Experiment 4. However, in this experiment the contagion information only had a statistically significant effect in the condition in which it was presented four times.

The main effect of item expectancy apparent in the data in Table 6 was also significant $\left[F(1,14)=12.62, M S_{\mathrm{e}}=\right.$ 0.06 ], with greater false recall for high- $(M=.28)$ than for low- $(M=.11)$ expectancy items. Low- and high-expectancy items were also examined in relation to number of presentations. The difference in the mean proportion of false recall for low-expectancy items presented four times $(M=.30)$ was significantly greater than the mean pro- portion of false recall for low-expectancy items presented zero times $(M=.03)[t(14)=3.23, S E M=.08]$, and one time $(M=.00)[t(14)=3.67, S E M=.08]$. For the highexpectancy items, the only significant difference in the mean proportion of false recall was between the contagion items presented four times $(M=.46)$ and the contagion items presented zero times $(M=.10)[t(14)=4.04, S E M=$ .09]. Although the data from the single-presentation condition fell between these two points $(M=.30)$, neither difference between the single-presentation condition and the other two conditions was statistically significant.

Analyses were also performed on the remember/know response data. As can be seen in Table 6, subjects were much more likely to give a know response to falsely recalled contagion items $(M=.19$, collapsing across one and four presentations and high- and low-expectancy items) than they were to give a remember response to falsely recalled contagion items $(M=.07)[t(14)=2.08$, $S E M=.06]$. This same pattern was not obtained for control items $(M=.05$ for know responses and $M=.02$ for remember responses) [ $t(14)=.81, S E M=.04, p>.05]$, perhaps because of a floor effect associated with low levels of responding.

Remember and know responses were analyzed separately, with data collapsed across one and four presentations of the contagionitems. In Experiments 1 and 2, there were only two conditions (contagion or control), so the six items in the contagion condition were divided into only high- and low-expectancy conditions. In Experiment 3, those six items in the contagion condition were not only split into high- and low-expectancy, but also into zero, one, and four presentations. This reduced the number of observations per cell and thus necessitated combining the contagion items in the one and four presentations for the remember/know analyses.

When remember responses were analyzed, a 2 (remember responses for contagion items) $\times 2$ (high- or lowexpectancy condition) ANOVA revealed a significant main effect only for the latter variable $[F(1,14)=9.95$, $\left.M S_{\mathrm{e}}=0.09\right]$. High-expectancy suggestions were more likely to be remembered than were low-expectancy items. Know responses were analyzed in the same manner, and significant main effects were obtained for both contagion $\left[F(1,14)=19.64, M S_{\mathrm{e}}=0.02\right]$ and expectancy $[F(1,14)=$ $\left.4.85, M S_{\mathrm{e}}=0.02\right]$. Subjects were more likely to give know responses for contagion than control items, and for highexpectancy relative to low-expectancy items. The interaction between factors was not significant.

Table 6

Experiment 3: Mean Proportion of False Recall and Remember or Know Responses for Low- and High-Expectancy Items Presented Zero, One, or Four Times

\begin{tabular}{|c|c|c|c|c|c|c|}
\hline \multirow[b]{3}{*}{ Response Type } & \multicolumn{6}{|c|}{ Number of Presentations } \\
\hline & \multicolumn{2}{|c|}{ Zero } & \multicolumn{2}{|c|}{ One } & \multicolumn{2}{|c|}{ Four } \\
\hline & Low Expect & High Expect & Low Expect & High Expect & Low Expect & High Expect \\
\hline Recall & .03 & .10 & .00 & .30 & .30 & .46 \\
\hline Remember & .00 & .03 & .00 & .13 & .03 & .13 \\
\hline Know & .03 & .07 & .00 & .17 & .27 & .33 \\
\hline
\end{tabular}


Table 7

Experiment 3: Mean Proportion of False and Veridical Source Judgments for Contagion and Control Items

\begin{tabular}{lccc}
\hline & \multicolumn{3}{c}{ Number of Presentations } \\
\cline { 2 - 4 } & Zero & One & Four \\
\hline Slide only & .30 & .07 & .00 \\
Both slide and confederate & .05 & .38 & .45 \\
Total false recognition & .35 & .45 & .45 \\
Confederate only & .08 & .35 & .50 \\
Neither slide nor confederate & .57 & .20 & .03 \\
\hline
\end{tabular}

The mean proportions of subjects' responses on the final recognition/source-monitoring test are displayed in Table 7, combined across item expectancy. As in Experiments 1 and 2 , we defined false recognition on the final recognition test as the proportion of items subjects recognized as having occurred in the scene (scene only) plus the proportion of items that subjects recognized both as occurring in the scene and as having been suggested by the confederate (both sources). Subjects falsely recognized a mean proportion of .45 of the contagion items (averaged across one and four presentations) compared with .35 of control items. This difference was marginally significant $[t(14)=2.04$, SEM $=$ $.05, p=.06]$. As in previous experiments, the surprise in these data is the high level of false recognition for the control items $(M=.35)$, items that had not been suggested by the confederate protocols. Again, we believe this outcome to be due to the high association between the contagion items and the other items in the scenes (Miller \& Gazzaniga, 1998; Roediger \& McDermott, 1995).

Finally, subjects correctly recalled a mean proportion of .37 items. This mean is the average of correct recall across scenes that ranged from .20 to .49 . The mean proportion of veridical recognition was .74 and ranged across scenes from .53 to .89 .

\section{EXPERIMENT 4}

The results of Experiment 3 raise an interesting issue regarding the use of an actual and an implied social pres- ence in the social contagion paradigm. Experiment 3 suggests that the effect can be obtained through the use of an implied social presence, but the effect may not be as strong. Is a response delivered by a virtual confederate weaker than that of an actual confederate, since the response of a single virtual confederate did not have an effect in Experiment 3 but did in the earlier experiments? However, before reaching this conclusion, one must consider several other procedural differences between experiments. In Experiments 1 and 2, the confederate and subject took turns giving responses, which may have permitted subjects to devote greater attention to each confederate response than the method of delivery used in Experiment 3, in which confederate responses were placed in a recall protocol. The purpose of Experiment 4 was to provide a direct comparison of the two types of social influence in a single experiment with other factors held constant.

\section{Method}

Subjects. The subjects were 24 Washington University undergraduates, who participated in the experiment for partial fulfillment of a course requirement.

Design. The experiment consisted of a $2 \times 2 \times 2$ mixed design. Exposure to social contagion (contagion or no contagion) and the expectancy of the contagion items (high expectancy or low expectancy) were manipulated within subjects, whereas the source of the contagion items (confederate or protocol) was manipulated between subjects. The dependent variables were false recall and false recognition of the suggested items.

Materials. The same basic materials used in earlier experiments were again employed in Experiment 4. Additional materials included the protocols of virtual confederates. These were index cards containing handwritten representations of the same responses given by the actual confederate in the other condition.

Procedure. For subjects who received the contagion items through a confederate, the procedure of Experiment 4 was identical to that of Experiment 2. For subjects who received the contagion items through a protocol, the procedure was identical to that of Experiment 2 except for the collaborative recall phase. For the collaborative recall phase, subjects were informed that this was the fourth of four studies in a series of experiments and that the protocols contained responses given by subjects who had participated in previous stages of the experiment. Subjects were then asked to take turns with the protocols in recalling items from each of the scenes. They were

Table 8

Experiment 4: Mean Proportion of False Recall and Remember or Know Responses for Low- and High-Expectancy Items When Misinformation was Presented by Confederate and Protocol

\begin{tabular}{|c|c|c|c|c|c|}
\hline \multirow{2}{*}{$\begin{array}{c}\text { Response } \\
\text { Type }\end{array}$} & \multicolumn{2}{|c|}{ Confederate } & \multicolumn{2}{|c|}{ Protocol } & \multirow[b]{2}{*}{$M$} \\
\hline & Low Expect & High Expect & Low Expect & High Expect & \\
\hline \multicolumn{6}{|c|}{ Contagion Items } \\
\hline Recall & .28 & .44 & .31 & .50 & .38 \\
\hline Remember & .08 & .16 & .03 & .11 & .09 \\
\hline Know & .20 & .28 & .28 & .39 & .29 \\
\hline \multicolumn{6}{|c|}{ Control Items } \\
\hline Recall & .03 & .14 & .00 & .22 & .10 \\
\hline Remember & .00 & .03 & .00 & .00 & .01 \\
\hline Know & .03 & .11 & .00 & .22 & .09 \\
\hline \multicolumn{6}{|c|}{ Difference } \\
\hline Recall & .25 & .30 & .31 & .28 & .28 \\
\hline Remember & .08 & .13 & .03 & .11 & .08 \\
\hline Know & .17 & .17 & .28 & .17 & .20 \\
\hline
\end{tabular}


Table 9

Experiment 4: Mean Proportion of False and Veridical Source Judgments for Contagion and Control Items

Contagion Items Control Items

Confederate

\begin{tabular}{lll} 
Slide only & .15 & .39 \\
Both slide and confederate & .55 & .08 \\
$\quad$ Total false recognition & .70 & .47 \\
Confederate only & .17 & .00 \\
Neither slide nor confederate & .13 & .53 \\
& Protocol & \\
Slide only & .20 & .46 \\
Both slide and protocol & .29 & .04 \\
$\quad$ Total false recognition & .49 & .50 \\
Protocol only & .35 & .00 \\
Neither slide nor protocol & .13 & .50 \\
\hline
\end{tabular}

instructed to recall one item aloud and then to turn over and silently read one index card so that the procedure would mimic the procedure in the other condition, where subjects and the confederate took turns recalling items from each of the scenes. In this way, the actual and virtual confederate conditions were better matched on the manner in which the suggested items were delivered. All other aspects of the experiment were like those of Experiment 2.

\section{Results and Discussion}

Table 8 gives the mean proportion of high- and lowexpectancy items falsely recalled when the contagion items were presented by the confederate and by the protocol. A 2 (contagion or no contagion) $\times 2$ (high-expectancy or low-expectancy) $\times 2$ (confederate or protocol) ANOVA revealed a significant main effect of contagion $[F(1,22)=$ $\left.30.13, M S_{\mathrm{e}}=0.01\right]$. Subjects were much more likely to falsely recall the contagionitems when they had been suggested $(M=.38)$ than when they had not been suggested $(M=.10)$. There was also a main effect of expectancy $\left[F(1,22)=25.84, M S_{\mathrm{e}}=0.03\right]$, showing that the social contagion effect was greater for high-expectancy items $(M=.33)$ than for low-expectancy items $(M=.16)$. These means are collapsed across contagion and control items and confederate and protocol conditions.

The main effect of source of the contagion items was not significant $\left[F(1,22)=.51, M S_{\mathrm{e}}=0.06\right]$, nor did this factor interact with the others. The social contagion effect was not significantly different for subjects in the confederate condition $(M=.28)$ than for subjects in the protocol condition $(M=.30)$. This pattern of results suggests that the power of a virtual confederate can be as great as that of an actual confederate on false recall when both methods alternate responses between the confederate and the subject so that the items produced by the confederate/ protocol are distinct and a focus of attention.

The mean proportions of remember and know responses are also displayed in Table 8. Again, the results of Experiment 4 revealed that subjects were more likely to give falsely recalled contagion items a know response $(M=$ .29 , collapsed across confederate and protocol conditions) than a remember response $(M=.09)[t(23)=3.52, S E M=$ $.05]$. This same pattern was also obtained for control items
( $M=.09$ for know responses and $M=.01$ for remember responses) $[t(23)=3.73, S E M=.02]$.

Remember and know responses were also analyzed separately to determine how they related to contagion, expectancy, and source of the contagion items. For remember responses, a 2 (remember responses for contagion or control items) $\times 2$ (high- or low-expectancy) $\times 2$ (confederate or protocol) ANOVA revealed a significant main effect for contagion items $\left[F(1,22)=9.94, M S_{\mathrm{e}}=0.02\right]$; a greater proportion of remember responses were given for suggesteditems $(M=.09)$ than for control items $(M=.01)$. This outcome indicates that the process of social contagion did influence subjects' reports of what they remembered from the scenes. Subjects were also more likely to give remember responses for high- than for low-expectancy items $\left[F(1,22)=5.02, M S_{\mathrm{e}}=0.01\right]$. Finally, the main effect of the source of contagion (actual vs. virtual confederate) was not significant $\left[F(1,22)=1.83, M S_{\mathrm{e}}=0.02, p>.05\right]$; subjects were equally likely to give a remember response when the items had been suggested by the confederate and when the items had been suggested by the protocol.

Know responses were analyzed across conditions in the same way. Again, there was a significant main effect for contagion $\left[F(1,22)=16.15, M S_{\mathrm{e}}=0.06\right]$, with subjects more likely to give a know judgment to the items that had been suggested $(M=.29)$ than to those same items when they had not been suggested $(M=.09)$. In addition, highexpectancy items were more likely to be given a know judgment than were low-expectancy items $[F(1,22)=$ $\left.15.49, M S_{\mathrm{e}}=0.02\right]$. Finally, there was no difference in the proportion of know responses when the items were suggested by the confederate and when they were suggested by the protocol $\left[F(1,22)=2.56, M S_{\mathrm{e}}=0.05, p>.05\right]$.

Subjects' performance on the recognition/sourcemonitoring test also resulted in significant levels of social contagion, as can be seen in Table 9. False recognition was again defined as the proportion of times subjects attributed the source of the item to the scene (this included both the slide-only category and the slide-plus-other-subject category on the final test). Interestingly, subjects who were presented with the contagion information via an actual confederate had higher rates of false recognition $(M=.23$, subtracting control from contagion items) than subjects who received the contagion information from nonhuman protocols $(M=-.01)[t(22)=2.35, S E M=.07]$. In addition, the proportions of responses comprising the false recognition score (slide-only vs. slide-plus-confederate) are markedly different for the confederate and protocol conditions. Specifically, subjects in the confederate condition were much more likely to attribute the source of the contagion items to the slide-plus-confederate category $(M=.47$, subtracting control from contagion proportions) than were subjects in the protocol condition $(M=.25)[t(22)=2.30$, $S E M=0.09]$. Perhaps the live person provided a more salient source in the recognition/source-monitoring test, and there may have been influences of credibility as well.

Finally, subjects correctly recalled a mean proportion of . 30 items across the six scenes. Veridical recall propor- 
tions ranged from .19 to .37 . The mean proportion of correct recognition was .75 and ranged from .56 to .92 .

\section{GENERAL DISCUSSION}

The four experiments reported here both replicate the social contagion effect reported by Roediger et al. (2001) and yield several interesting new findings. Perhaps the most important new finding, obtained in all four experiments, is that the social contagion effect occurs on a recognition/source-monitoring test in which subjects' attention is drawn to the possible source of the misinformation. Because a source-monitoring test reduces or even eliminates the standard misinformation effect (e.g., Lindsay \& Johnson, 1989) and the false fame effect reported by Jacoby, Kelley, Brown, and Jasechko (1989; also see Multhaup, 1995), it is noteworthy that social contagion is a powerful enough phenomenon to persist even under these stringent testing conditions. Evidence of the social contagion effect persisted on a source-monitoring test in spite of an explicit warning (Experiment 1) and regardless of whether or not the test was preceded by recall tests covering the same information (Experiment 2).

Several other new findings were obtained in our experiments. Speeding presentation rate of the slides from 15 to $5 \mathrm{sec}$ per slide did not increase the social contagion effect (Experiment 2). However, increasing the number of presentations of the misinformation did enhance the effect (Experiment 3). We also showed that when care is taken to equate the manner of presentation between the conditions, the impact of the false recollection of an actual confederate was the same as that delivered by a virtual confederate on a recall test, but not on a source-monitoring test (Experiment 4). Of course, this last result, which is provocative, bears replication in future experiments.

\section{Relation to Other Paradigms}

The social contagion paradigm blends the conformity paradigm of Asch (1956) and the misinformation paradigm of Loftus (1979). This fact led us to ask whether variables with known effects on those paradigms would generalize to our situation. The answer is both yes and no. We did find that, as in the conformity and the misinformation paradigms (Asch, 1956; Zaragoza \& Mitchell, 1996), increasing the number of erroneous responses increased the magnitude of the effect (Experiments 3 and 4). However, unlike results in the conformity paradigm, the changes in subjects' responses seem to reflect private acceptance (changes in belief or reports of what is recollected), not just in public acceptance and public reporting. We feel confident in making this claim because (1) subjects were tested in private on the critical test, with a premium placed on accurate recollection; (2) in most of our experiments the social contagion effect occurred on remember responses as well as on know responses; and (3) the social contagion effect also occurred in a sourcemonitoring test that explicitly called subjects' attention to the various possible sources of information and permitted them to select the most appropriate categories. Thus, as in the misinformation effect (see Weingardt et al., 1994), subjects change not just their reports, but the beliefs underlying those reports.

The issue of whether responses of actual confederates have greater power to reshape the subject's memories than do responses allegedly written by other subjects (virtual confederates) was examined here in Experiments 3 and 4. In Experiment 3 we found that responses of virtual confederates did produce the social contagion effect (also see Betz et al., 1996, and Schneider \& Watkins, 1996), but to a lesser extent than the actual confederates in Experiments 1 and 2. However, in Experiment 3, the virtual confederates' responses were delivered in a long list, whereas in the earlier experiments the actual subject took turns with the confederate in providing responses, which probably made the confederate's responses more salient. In Experiment 4, we made this feature of the procedure more similar between conditions by having the responses of the virtual confederate delivered one at a time, as were those of the actual confederate. We now found that the social contagion effect was not significantly different in cued recall between these two conditions, but that on the source monitoring test the effect of social contagion was greater for the actual than for the virtual confederate. A recent study by Kelley, Sahakyan, and Rhodes (2001) also revealed some interesting differences between the influence of an actual and a virtual confederate on estimates of memory and conformity bias. Of course, this pattern of results needs further replication, but if confirmed our findings would represent the relatively rare case of a false memory effect involving a confusion of sources appearing on a source-monitoring test when it does not appear on a cued-recall test.

One interesting feature of the present experiments is the relatively high rates of false recall and false recognition in the control condition, especially for the expected or typical items. For example, averaging across the two presentation durations of Experiment 2, subjects recalled the expected or typical items as having been in the scene on $22 \%$ of the trials in the control condition; the false alarm rate from the recognition/source-monitoring test was $48 \%$. We believe these results reflect the fact that even the control condition used here potentiates large false memory effects that resemble those in paradigms in which scene schemas are strongly activated (e.g., Brewer \& Treyens, 1981; Miller \& Gazzaniga, 1998) or that contain highly interassociated items (Roediger \& McDermott, 1995). Because the high-expectancy items seem potentiated for arousal during encoding, the finding that these items are more easily incorporated as false memories (than are less expected items) when suggested by the confederate is not surprising and fits well with virtually all theories of how false memories develop. We turn now to consider theoretical issues.

\section{Theoretical Implications}

Our aim was more methodological than theoretical; we wanted to develop a blend of the conformity and misinformation paradigms. We describe below an account of our result in terms of Johnson's source-monitoring frame- 
work for understanding false memories (e.g., Johnson et al., 1993), although we hasten to add that other theories are certainly not excluded by our data. We find the sourcemonitoring framework a congenial perspective and one that is fruitful in suggesting future research.

According to the source-monitoring framework, subjects fall prey to misinformation because they have confused the source of the original information with the source of the misinformation (e.g., Johnson et al., 1993; Lindsay, 1994; Lindsay \& Johnson, 1989). Similarly, subjects in the social contagion paradigm may become confused about whether they are recalling an item because they actually saw it or because the confederate suggested it. If the suggested item (e.g., a toaster) comes to mind during the test, its familiarity may be misattributed to the scene rather than accurately attributed to the confederate's response (Jacoby, Kelley, \& Dywan, 1989). This process of incorporating the erroneous information into recollections of the scene may be enhanced by the fact that the confederate's response came during his/her alleged recollection of the scene itself.

Source misattributions are most likely to occur when the possible sources of information are similar (e.g., Johnson, Foley, \& Leach, 1988; Johnson \& Raye, 1998). Johnson and Raye suggested that source misattribution errors increase when items are perceptually similar (e.g., Koutstaal \& Schacter, 1997) as well as when items are semantically similar (e.g., Roediger \& McDermott, 1995). In the social contagion paradigm, the high degree of semantic relatedness between the suggested items and the items that actually were present in the scenes may have contributed to subjects' source confusions. This conjecture is supported by the finding that false recall in all four experiments (and in Roediger et al.'s, 2001, experiment) was greater for high- than for low-expectancy items.

The source-monitoring framework also accounts well for the warning data obtained in Experiment 1. The fact that the warnings reduced the social contagion effect but did not eliminate it suggests that instructions to subjects to carefully monitor their reports do have some effect. When subjects are told to be careful to retrieve only accurate information, they are better able to disregard the inaccurate information and the incidence of false memory decreases. However, Experiment 1 suggested that the reduction in false memories reflected a general criterion shift that affected all responses given by the confederate, not just those that were erroneous.

The results of the recognition/source-monitoring tests revealed that subjects were relatively good at correctly attributing spoken items to the confederate, although they often erroneously believed these items had also appeared in the scenes. This outcome with regard to source replicates past research in the misinformation paradigm showing that subjects are able to differentiate between original sources and sources of misinformation (e.g., Zaragoza \& Koshmider, 1989). Yet despite memory of this information for specific features of presentation, the illusion that the item also appeared in the scene persisted quite strongly, by appearing on source-monitoring tests of the sort that reduce or eliminate other memory illusions. Although items produced by the confederate are probably quite distinct (and hence memorable) relative to the numerous other items that were seen in the slides, the illusion nonetheless persists. This outcome shows the powerful influence that social factors, such as suggestions by others, may have on memory reports and also show that one person's erroneous responses can infect another person's memory.

We end with a disclaimer. The social contagion language we have used throughout this paper-assuming that memories are infectious, like some diseases-represents only a partial truth. The recollections of one person can also have beneficial effects on recollections of another. If two people witness a scene and if, in later joint recollection, one person cannot recall details that are confidently recalled by anotherperson, then it would be adaptive for the person with the poorer memory to update his/her memory with the other person's fuller account. The source of the social contagion effect may represent this adaptive process gone awry when the suggested information is less than veridical. However, social processes may normally serve to enhance rather than to undermine another person's recollections.

\section{REFERENCES}

Allen, V. L. (1965). Situational factors in conformity. In L. Berkowitz (Ed.), Advances in experimental social psychology (Vol. 2, pp. 133175). Oxford: Academic Press.

Asch, S. E. (1956). Studies of independence and conformity: A minority of one against a unanimous majority. Psychological Monographs, 70, 416.

Ayers, M. S., \& Reder, L. M. (1998). A theoretical review of the misinformation effect: Predictions from an activation-based memory model. Psychonomic Bulletin \& Review, 5, 1-21.

BARTLETT, F. C. (1932). Remembering: A study in experimental and social psychology. New York: Cambridge University Press.

Betz, A. L., Skowronski, J. J., \& Ostrom, T. M. (1996). Shared realities: Social influence and stimulus memory. Social Cognition, 14, 113-140.

Binet, A. (1900). La suggestibilitè. Paris: Schleicher Frères.

BREWER, W. F. (1977). Memory for the pragmatic implications of sentences. Memory \& Cognition, 5, 673-678.

Brewer, W. F., \& Treyens, J. C. (1981). Role of schemata in memory for places. Cognitive Psychology, 13, 207-230.

CECI, S. J., \& BRUCK, M. (1995). Jeopardy in the courtroom: A scientific analysis of children's testimony. Washington, DC: American Psychological Association.

Ceci, S. J., Ross, D. F., \& Toglia, M. P. (1987). Suggestibility of children's memory: Psycholegal implications. Journal of Experimental Psychology: General, 116, 38-49.

Deutsch, M., \& Gerard, H. B. (1955). A study of normative and informative social influences upon individual judgment. Journal of $A b$ normal \& Social Psychology, 51, 629-636.

FAncher, R. E. (1996). Pioneers of psychology. New York: Norton. Gallo, D. A., Roberts, M. J., \& Seamon, J. G. (1997). Remembering words not presented in lists: Can we avoid creating false memories? Psychonomic Bulletin \& Review, 4, 271-276.

Gallo, D. A., \& Roediger, H. L., III (2002). Variability among word lists in evoking associative memory illusions. Journal of Memory \& Language, 47, 469-497.

Gallo, D. A., Roediger, H. L., III, \& McDermott, K. B. (2001). Associative false recognition occurs without strategic criterion shifts. Psychonomic Bulletin \& Review, 8, 579-586.

GARDINER, J. M. (1988). Functional aspects of recollective experience. Memory \& Cognition, 16, 309-313.

Hoffman, H. G., Granhag, P. A., Kwong See, S. T., \& Loftus, E. F. (2001). Social influences on reality-monitoring decisions. Memory \& Cognition, 29, 394-404. 
Jacoby, L. L., Kelley, C. M., Brown, J., \& Jasechko, J. (1989). Becoming famous overnight: Limits on the ability to avoid unconscious influences of the past. Journal of Personality \& Social Psychology, 56, 326-338.

Jacoby, L. L., Kelley, C. M., \& Dywan, J. (1989). Memory attributions. In H. L. Roediger III \& F. I. M. Craik (Eds.), Varieties of memory and consciousness: Essays in honour of Endel Tulving (pp. 391422). Hillsdale, NJ: Erlbaum.

Johnson, M. K., Foley, M. A., \& LeACH, K. (1988). The consequences for memory of imagining in another person's voice. Memory \& Cognition, 16, 337-342.

Johnson, M. K., Hashtroudi, S., \& Lindsay, D. S. (1993). Source monitoring. Psychological Bulletin, 114, 3-28.

Johnson, M. K., \& RAYE, C. L. (1998). False memories and confabulation. Trends in Cognitive Sciences, 2, 137-145.

Kelley, C. M., Sahakyan, L., \& Rhodes, M. G. (2001, November). Social conformity in memory. Poster presented at the annual meeting of the Psychonomic Society, Orlando.

Kelman, H. C. (1961). Processes of opinion change. Public Opinion Quarterly, 25, 57-78.

Koutstaal, W., \& Schacter, D. L. (1997). Gist-based false recognition of pictures for older and younger adults. Journal of Memory \& Language, 37, 555-583.

LiNDSAY, D. S. (1994). Memory source monitoring and eyewitness testimony. In D. F. Ross, J. D. Read, \& M. P. Toglia (Eds.), Adult eyewitness testimony: Current trends and developments (pp. 27-55). New York: Cambridge University Press.

LindSAY, D. S., \& Johnson, M. K. (1989). The eyewitness suggestibility effect and memory for source. Memory \& Cognition, 17, 349-358.

LofTus, E. F. (1979). Eyewitness testimony. Cambridge, MA: Harvard University Press.

Loftus, E. F., Miller, D. G., \& Burns, H. J. (1978). Semantic integration of verbal information into a visual memory. Journal of Experimental Psychology: Human Learning \& Memory, 4, 19-31.

Loftus, E. F., \& Palmer, J. C. (1974). Reconstruction of automobile destruction: An example of the interaction between language and memory. Journal of Verbal Learning \& Verbal Behavior, 13, 585-589.

LuUs, C. A. E., \& Wells, G. L. (1994). The malleability of eyewitness confidence: Co-witness and perseverance effects. Journal of Applied Psychology, 79, 714-723.

McDermott, K. B., \& Roediger,H. L., III (1998). Attempting to avoid illusory memories: Robust false recognition of associates persists under conditions of explicit warnings and immediate testing. Journal of Memory \& Language, 39, 508-520.

McDermott, K. B., \& Watson, J. M. (2001). The rise and fall of false recall: The impact of presentation duration. Journal of Memory \& Language, 45, 160-176.

Miller, M. B., \& Gazzaniga, M. S. (1998). Creating false memories for visual scenes. Neuropsychologia, 36, 513-520.

Mitchell, K. J., \& ZaragOZA,M. S. (1996). Repeated exposure to suggestion and false memory: The role of contextual variability. Journal of Memory \& Language, 35, 246-260.

Multhaup, K. S. (1995). Aging, source, and decision criteria: When false fame errors do and do not occur. Psychology \& Aging, 10, $492-497$.
RAJARAM, S. (1993). Remembering and knowing: Two means of access to the personal past. Memory \& Cognition, 21, 89-102.

RoEdiger, H. L., III (1996). Memory illusions. Journal of Memory \& Language, 35, 76-100.

Roediger, H. L., III, JACoby, J. D., \& McDermott, K. B. (1996). Misinformation effects in recall: Creating false memories through repeated retrieval. Journal of Memory \& Language, 35, 300-318.

RoEdiger, H. L., III, \& MCDermott, K. B. (1995). Creating false memories: Remembering words not presented in lists. Journal of Experimental Psychology: Learning, Memory, \& Cognition, 21, 803-814.

Roediger, H. L., III, \& McDermott, K. B. (2000). Distortions of memory. In E. Tulving \& F. I. M. Craik (Eds.), The Oxford handbook of memory (pp. 149-162). New York: Oxford University Press.

Roediger, H. L., III, Meade, M. L., \& Bergman, E. T. (2001). The social contagion of memory. Psychonomic Bulletin \& Review, 8, 365371.

SCHACTER, D. L. (1995). Memory distortion: History and current status. In D. L. Schacter, J. T. Coyle, G. D. Fischbach, M. M. Mesulam, \& L. E. Sullivan (Eds.), Memory distortion (pp. 1-43). Cambridge, MA: Harvard University Press.

Schneider, D. M., \& WATKINS, M. J. (1996). Response conformity in recognition testing. Psychonomic Bulletin \& Review, 3, 481-485.

Schreiber, T. A., \& SErgent, S. D. (1998). The role of commitment in producing misinformation effects in eyewitness memory. Psychonomic Bulletin \& Review, 5, 443-448.

ShaW, J. S., III, GaRven, S., \& Wood, J. M. (1997). Co-witness information can have immediate effects on eyewitness memory reports. Law \& Human Behavior, 21, 503-523.

Toglia, M. P., Neuschatz, J. S., \& Goodwin, K. A. (1999). Recall accuracy and illusory memories: When more is less. Memory, 7, 233-256.

Tulving, E. (1985). Memory and consciousness. Canadian Psychologist, 26, 1-12.

Weingardt, K. R., Toland, H. K., \& Loftus, E. F. (1994). Reports of suggested memories: Do people truly believe them? In D. F. Ross, J. D. Read, \& M. P. Toglia (Eds.), Adult eyewitness testimony: Current trends and developments (pp. 3-26). New York: Cambridge University Press.

WELDON, M. S. (2000). Remembering as a social process. In D. L. Medin (Ed.), The psychology of learning and motivation (Vol. 40, pp. 67120). San Diego: Academic Press.

Whipple, G. M. (1909). The observer as reporter: A survey of the psychology of testimony. Psychological Bulletin, 6, 153-170.

WRIGHT, D. B. (1993). Misinformation and warnings in eyewitness testimony: A new testing procedure to differentiate explanations. Memory, 1, 153-166.

Wright, D. B., Self, G., \& Justice, C. (2000). Memory conformity: Exploring misinformation effects when presented by another person. British Journal of Psychology, 91, 189-202.

Zaragoza, M. S., \& Koshmider, J. W. (1989). Misled subjects may know more than their performance implies. Journal of Experimental Psychology: Learning, Memory, \& Cognition, 15, 246-255.

Zaragoza, M. S., \& Mitchell, K. J. (1996). Repeated exposure to suggestion and the creation of false memories. Psychological Science, 7 , 294-300.

\section{APPENDIX \\ High- and Low-Expectancy Contagion Items (With Alternates in Parentheses) for Six Scenes}

\begin{tabular}{lll} 
Scene & High Expectancy & \multicolumn{1}{c}{ Low Expectancy } \\
\hline Toolbox & screws (pliers) & ruler (pencil) \\
Bathroom & toothbrush(soap) & hair brush(lens solution) \\
Kitchen & toaster (knife) & oven mitts (napkins) \\
Bedroom & clock (mirror) & cologne (night lamp) \\
Closet & shoes (boxes) & belt (ball) \\
Desk & printer(stapler) & rolodex (calendar) \\
\hline
\end{tabular}

